\title{
Higher Rates of Persistence and Adherence in Patients with Type 2 Diabetes Initiating Once-Weekly vs Daily Injectable Glucagon-Like Peptide-1 Receptor Agonists in US Clinical Practice (STAY Study)
}

\author{
William H. Polonsky (D) · Riya Arora · Mads Faurby · João Fernandes • \\ Andreas Liebl
}

Received: October 7, 2021 / Accepted: November 29, 2021 / Published online: December 16, 2021

(C) The Author(s) 2021

\section{ABSTRACT}

Introduction: Reducing dosing frequency may lower treatment burden and improve persistence and adherence. This retrospective, observational study assessed persistence and adherence in patients with type 2 diabetes (T2D) initiating once-weekly or daily injectable glucagon-like peptide-1 receptor agonists (GLP-1 RAs) in US clinical practice.

Methods: The study used data from adults ( $\geq 18$ years) with T2D who were included in the IBM MarketScan Explorys Claims-EMR Data Set for $\geq 180$ days pre-index and $\geq 365$ days post-

Supplementary Information The online version contains supplementary material available at https:// doi.org/10.1007/s13300-021-01189-6.

W. H. Polonsky $(\bowtie)$

Behavioral Diabetes Institute, 5230 Carroll Canyon

Road, Suite 208, San Diego, CA 92121, USA

e-mail: whp@behavioraldiabetes.org

W. H. Polonsky

University of California, San Diego, CA, USA

R. Arora

Novo Nordisk Service Centre Pvt Ltd, Bangalore, India

M. Faurby $\cdot$ J. Fernandes

Novo Nordisk A/S, Søborg, Denmark

A. Liebl

Center for Diabetes and Metabolism, m\&i-

Fachklinik Bad Heilbrunn, Bad Heilbrunn, Germany index, were GLP-1 RA and insulin naïve at first claim (index date) for once-weekly or daily injectable GLP-1 RAs (follow-up: index date +365 days), and were propensity score (PS) matched 1:1 by baseline characteristics. Persistence, defined as the stay time, was assessed using Kaplan-Meier analysis and Cox proportional hazards models. Adherence was defined as a proportion of days covered of 0.8 or greater. To assess whether patients with more advanced disease would benefit from long-acting treatments, patients were matched to the baseline characteristics of basal insulin initiators using inverse probability of treatment weighting (IPTW).

Results: The PS-matched cohorts $(n=784$ each) had similar baseline characteristics. Onceweekly regimens were associated with significantly higher persistence than daily treatments (median stay time: 333 vs 269 days; hazard ratio 0.80 [95\% confidence interval 0.71, 0.90]; $p<0.01)$ and with significantly higher adherence than daily regimens at 6 months and 12 months ( $p<0.01$ for both). Mean glycated haemoglobin reductions were greater with once-weekly than with daily treatment at 6 months $(-1.1 \%$ vs $-0.9 \% ; p<0.01)$ and 12 months $(-0.9 \%$ vs $-0.7 \%$; $p=$ not significant); adherent patients experienced greater reductions than those with poor adherence. Results were similar in the IPTW-matched analysis. 
Conclusion: In US clinical practice, onceweekly injectable treatments are associated with better persistence and adherence than daily regimens over 1 year. Once-weekly regimens may also benefit patients with more advanced T2D.

\section{PLAIN LANGUAGE SUMMARY}

For people who take medication over a prolonged time, less frequent doses are often more convenient. This can help people to continue with treatment for as long as prescribed (persistence) and to take their medication as prescribed (adherence). This study examined persistence and adherence in people with type 2 diabetes who started treatment with glucagonlike peptide-1 receptor agonists (GLP-1 RAs). The GLP-1 RAs in this study are injected under the skin using a small needle. Some types are given daily and others are given once weekly. We used a US database of medical records to identify people with type 2 diabetes who had been prescribed these medications and split them into two groups (784 people each) with similar characteristics. One group had received once-weekly GLP-1 RAs and the other group had received daily GLP-1 RAs. We found that the once-weekly group continued taking their medication for longer (333 days) than the daily group (269 days). People in the once-weekly group were also $20 \%$ less likely to stop the treatment too early than those in the daily group. More people in the once-weekly group took their medication as prescribed over 1 year compared with the daily group. The onceweekly group also had larger reductions in blood sugar levels after 1 year than the daily group. People who took their medication as prescribed had the greatest improvements in blood sugar levels. Our results suggest that GLP1 RAs that are injected less frequently can help people to take their medication as prescribed.

\section{Graphical abstract:}

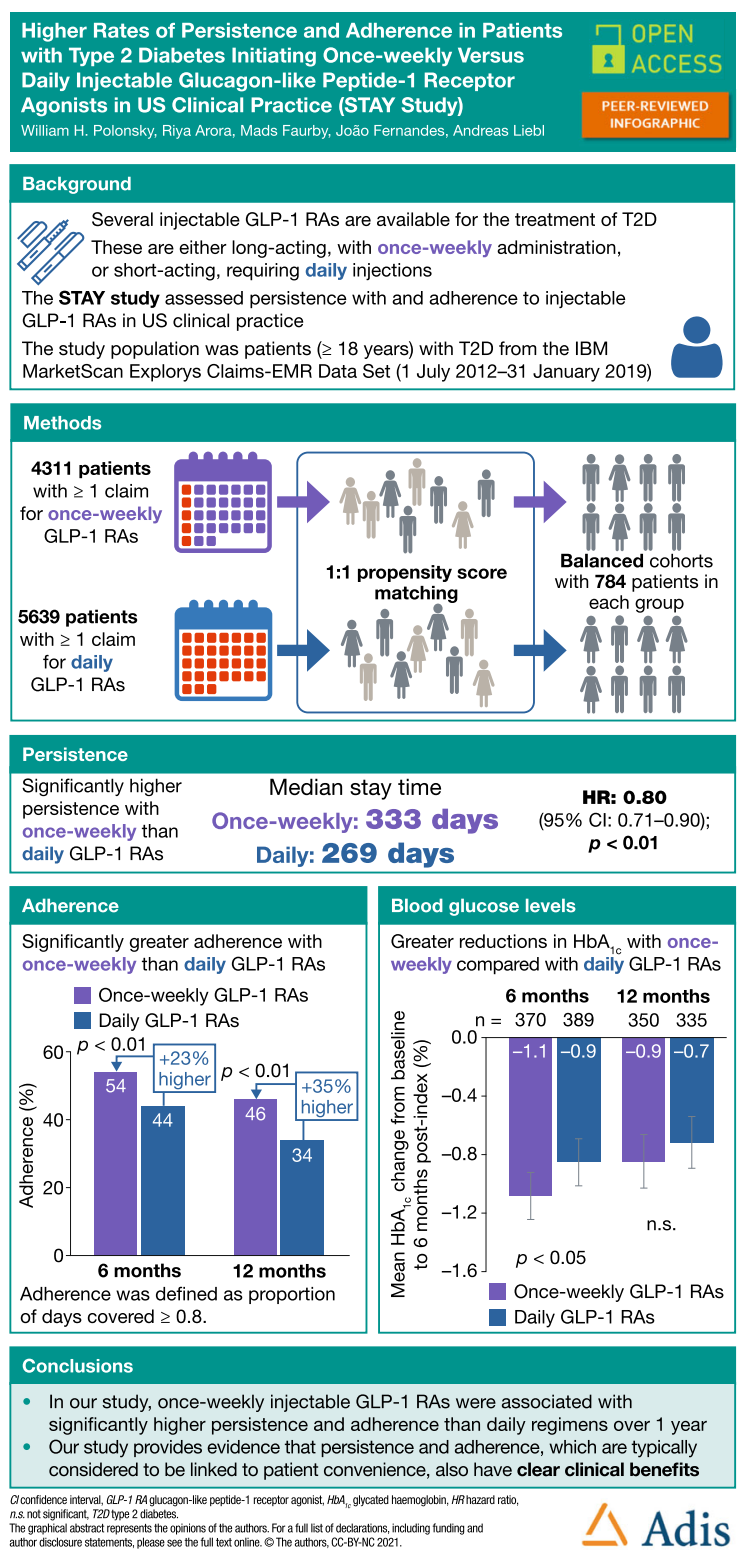

Keywords: Adherence; GLP-1 receptor agonist; Observational; Persistence; Real-world evidence; Type 2 diabetes 


\section{Key Summary Points}

\section{Why carry out this study?}

Medication persistence and adherence can confer a treatment benefit in type 2 diabetes (T2D), whereas poor persistence and adherence are linked to long-term complications, hospitalization and mortality.

The aim of this retrospective, observational study was to investigate persistence and adherence in patients with T2D initiating treatment with onceweekly or daily injectable glucagon-like peptide-1 receptor agonists (GLP-1 RAs) using US administrative claims data.

\section{What was learned from the study?}

In US clinical practice, persistence with and adherence to once-weekly GLP-1 RAs were significantly greater compared with those of daily GLP-1RAs.

Glycated haemoglobin (HbA1c) reductions were greater with once-weekly vs daily injectable treatments at both 6 and 12 months after initiation; for both treatment regimens, better adherence was associated with greater improvements in HbA1c.

Our study suggests that persistence and adherence, which are typically considered to be linked to patient convenience, also have clear clinical benefits.

\section{DIGITAL FEATURES}

This article is published with digital features, including a graphical abstract, to facilitate understanding of the article. To view digital features for this article go to https://doi.org/10. 6084/m9.figshare.17091752.

\section{INTRODUCTION}

Glucagon-like peptide-1 receptor agonists (GLP1 RAs) have been shown to improve glycaemic control and to promote weight loss in the treatment of type 2 diabetes (T2D), and are associated with a low risk of hypoglycaemia [1]. For the management of T2D, GLP-1 RAs are recommended as the first injectable therapy before basal insulin in national and international guidelines; the American Diabetes Association recommends GLP-1 RAs as a treatment option for patients not achieving glycaemic control with oral antidiabetic agents and for those with or at high risk of atherosclerotic cardiovascular disease (CVD) [2]. In addition, GLP-1 RAs should be considered in patients with a contraindication for or intolerance of metformin [2].

Various injectable GLP-1 RAs are available for the treatment of T2D. These include GLP-1 RAs that need to be administered on a daily basis (liraglutide, lixisenatide and exenatide immediate release) and GLP-1 RAs requiring weekly injections (semaglutide, dulaglutide and exenatide extended release) [1]. In regions including Europe and the USA, an oral formulation of semaglutide is also available [3, 4]. Factors influencing the choice of long-acting or shortacting GLP-1 RAs include efficacy, tolerability, convenience, comorbidities and patient preference $[2,5]$. In countries without universal healthcare, prescription drug out-of-pocket costs are an additional consideration [6]. The efficacy of injectable GLP-1 RAs has been demonstrated across a number of randomized controlled trials, such as the SUSTAIN clinical trial programme for semaglutide [7] and the LEAD clinical trial programme for liraglutide [8].

Medication persistence and adherence remain a challenge in $\mathrm{T} 2 \mathrm{D}$ care. Treatment benefit is thought to be linked to both persistence and adherence, and poor persistence and adherence can increase the risks of long-term complications, hospitalization and mortality [9]. Increasing persistence and adherence is therefore an important goal in the treatment of patients with T2D. There is evidence that a 
more convenient administration schedule, for example via a reduced dosing frequency, offers a reduced treatment burden and improves medication persistence and adherence. A systematic review of 76 studies measuring compliance (defined as dose taking [taking the prescribed medication each day] and dose timing [taking medication within the prescribed time frame]) across a variety of therapeutic classes found that significantly higher compliance was associated with dosing regimens that require less frequent administration [10]. Similarly, higher persistence with and adherence to injectable GLP-1 RAs with less frequent dosing schedules have been reported. A database study examining treatment patterns across five European countries found that treatment persistence after 1 year was generally highest among patients initiating once-weekly GLP-1 RAs, followed by once-daily and twice-daily regimens [11].

Evaluating persistence and adherence in realworld populations of patients treated for T2D can provide important insights into both medication-taking behaviour and treatment benefit. In this observational, retrospective study, we assessed persistence and adherence, and their relationship with HbA1c levels and weight, in patients with T2D initiating once-weekly or daily injectable GLP-1 RAs in clinical practice using linked US electronic medical records (EMRs) and administrative claims data.

\section{METHODS}

\section{Data Source}

This study used data from individuals identified in the US IBM MarketScan Explorys Claims-EMR Data Set (IBM Watson Health, Armonk, NY, USA) [12], which integrates administrative claims from the MarketScan Commercial and Medicare Supplemental Databases with IBM Explorys EMRs. This is a commercial database, and the data set was accessed via a licence with IBM. It spans 40 million unique patients across all care settings, including physician visits, hospital stays and pharmacies [13]. Data are collated from more than 500 hospitals over 120 employers, more than 40 health plans and between nine and twelve Medicaid agencies, and comprise claims for inpatient and outpatient services and outpatient prescription drugs [13]. Patients represented in these datasets are disproportionately from the US South and Midwest, as discussed in previous studies $[14,15]$. The study was performed in accordance with the Helsinki Declaration of 1964 and its later amendments; approval by an ethics committee was not required because only de-identified secondary data were used.

\section{Study Design and Patient Population}

This was an observational, retrospective study (Fig. 1). Patients aged $\geq 18$ years with an International Classification of Diseases (ICD)-9 or ICD-10 code $[16,17]$ for T2D and at least one claim for an injectable once-weekly GLP-1 RA (semaglutide, dulaglutide or exenatide extended release) or daily injectable GLP-1 RA (liraglutide, lixisenatide, or exenatide immediate release) were included. Patients were required to be GLP-1 RA and insulin naïve at first claim. Patients with ICD-9/10 codes for type 1 diabetes, gestational diabetes or secondary diabetes, and those with a claim for insulin degludec/liraglutide or insulin glargine/lixisenatide, were excluded.

The index date was the date of the first claim for GLP-1 RA; the index period was 1 July 2012 to 31 January 2019, and the follow-up was the index date +365 days. Patients required $\geq 180$ days of continuous enrolment in the database pre-index and $\geq 365$ days of continuous enrolment in the database post-index.

\section{Outcomes}

The primary endpoint was persistence, defined as the stay time. Discontinuation (which marks the end of the stay time) was defined as $\geq 60$ days not covered by medication. In the sensitivity analysis, discontinuation was defined as $\geq 90$ days not covered by medication. For stay time, patients who had not discontinued within 12 months were censored at this time. The secondary endpoint was 


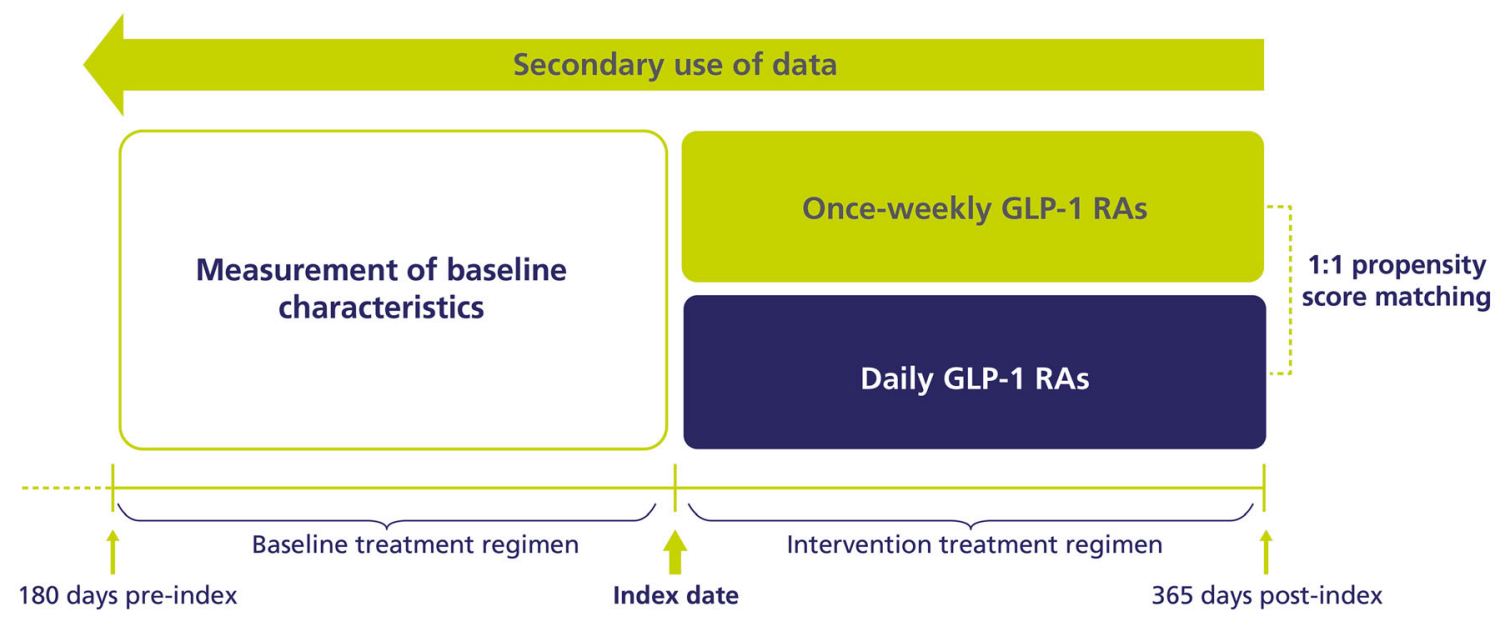

Fig. 1 Study design. GLP-1 $R A$ glucagon-like peptide-1 receptor agonist

adherence to treatment, defined as a proportion of days covered (PDC) of $\geq 0.8$. PDC is the number of days during a 6-month or 12-month period that the patient has a supply of their prescribed medication divided by the total number of days in that period. We also assessed the changes in glycated haemoglobin (HbA1c) and weight from baseline at 6 months and 12 months, determined from HbA1c and weight measurements within 45 days either side of the 6 months and 12 months post-index marks. For patients who had several available measurements, the one closest to the 6-month or 12-month mark was used, and if several measurements were recorded on the same date, the mean was used.

\section{Propensity Score Matching}

Propensity score (PS) matching allows subclasses to be analysed and compared in observational studies by balancing baseline characteristics across cohorts [18]. For the main analysis, patients treated with once-weekly or daily GLP-1 RAs were PS matched 1:1 by baseline age, sex, Charlson Comorbidity Index (CCI) score, HbA1c and weight (90 days pre-index) and use of sulfonylureas, metformin and dipeptidyl peptidase-4 inhibitors (180 days preindex). In a sensitivity analysis, previous CVD and use of sodium-glucose cotransporter-2 inhibitors (SGLT-2is) were included in the PS matching in addition to the baseline criteria above. A standardized mean difference (SMD) of $<0.1$ is indicative of a balanced cohort [19].

\section{Statistical Analyses}

Descriptive data for baseline characteristics are presented as the mean and standard deviation or the median for continuous variables and as the number and percentage for categorical variables. The two-tailed $t$-test was performed to compare continuous variables, and Fisher's exact test was performed for categorical variables. Kaplan-Meier analysis was used to assess time to treatment discontinuation, and a Cox proportional hazards model was used to estimate hazard ratios (HRs) to compare persistence; both analyses included all of the followup data.

\section{Secondary Analysis: Matching to Patients with Characteristics of Basal Insulin Initiators}

A secondary analysis was performed to assess whether patients at a later stage in the T2D treatment pathway and with more advanced disease, as expected for basal insulin initiators, would benefit from treatments that are potentially associated with better persistence and adherence. For this, patients treated with onceweekly or daily GLP-1 RAs were matched to the 
clinical and demographic characteristics of basal insulin initiators using inverse probability of treatment weighting (IPTW). IPTW matching was chosen over PS matching due to variations in the baseline characteristics between patients initiating therapy with GLP1-RAs and those initiating with basal insulin. Unlike PS matching, the IPTW method uses the whole dataset and reweighs individuals to increase the weights of those in the target population, from which there were few observations. This method creates a pseudo-population with nearperfect covariate balance between treatment groups, and allows the estimation of the treatment effect for the full cohort of patients matching the eligibility criteria [20, 21]. IPTW weights were assigned to patients in both cohorts, and GLP-1 RA categories were matched with those of basal insulin initiators; weights assigned were 1/PS for the insulin group and $1 /[1$ - PS] for the GLP-1 RA groups. The baseline characteristics used for IPTW matching were baseline age, sex, HbA1c and weight (90 days pre-index) and use of sulfonylureas, metformin, dipeptidyl peptidase- 4 inhibitors and SGLT-2is (180 days pre-index). An SMD of $<0.1$ is indicative of a balanced cohort [19].

\section{RESULTS}

\section{Study Population and Baseline Characteristics}

A total of 4311 patients receiving once-weekly injectable GLP-1 RAs and 5639 patients receiving daily injectable GLP-1 RAs, all of whom had T2D and met all the other inclusion criteria, were included in the analysis (Supplemental Table S1). Before matching, there were some disparities in baseline characteristics (Supplemental Table S2). Compared with the daily GLP1 RA cohort, the once-weekly GLP-1 RA cohort had a lower proportion of women $(49 \%$ vs $56 \%)$, and more patients were treated with SGLT-2is (10\% vs 4\%). Following PS matching, each of the GLP-1 RA cohorts included 784 individuals, and the matched cohorts had similar baseline characteristics, with SMD $<0.1$ for all characteristics (Table 1). GLP-1 RA use within cohorts is shown in Supplemental Table S3. Patients had a similar baseline mean age (onceweekly GLP-1 RAs: 55 years; daily GLP-1 RAs: 54 years), body mass index $\left(36.5 \mathrm{~kg} / \mathrm{m}^{2}\right.$; $36.7 \mathrm{~kg} / \mathrm{m}^{2}$ ) and CCI score (both 0.5). Similar

Table 1 Baseline characteristics of the PS-matched GLP-1 RA cohorts

\begin{tabular}{|c|c|c|c|}
\hline $\begin{array}{l}\text { Baseline } \\
\text { characteristics }\end{array}$ & $\begin{array}{l}\text { Once-weekly } \\
\text { GLP-1 RAs } \\
n=784\end{array}$ & $\begin{array}{l}\text { Daily } \\
\text { GLP-1 } \\
\text { RAs } \\
n=784\end{array}$ & SMD \\
\hline $\begin{array}{l}\text { Age, years, mean } \\
\text { (SD) }\end{array}$ & $54.6(9.9)$ & $\begin{array}{l}54.4 \\
\quad(10.2)\end{array}$ & 0.02 \\
\hline Women, $n(\%)$ & $392(50.0)$ & $\begin{array}{l}402 \\
\quad(51.3)\end{array}$ & 0.03 \\
\hline $\begin{array}{l}\text { Weight, kg, mean } \\
\text { (SD) }\end{array}$ & $107.0(23.7)$ & $\begin{array}{r}107.9 \\
(24.6)\end{array}$ & -0.04 \\
\hline $\begin{array}{l}\text { Body mass index, } \\
\mathrm{kg} / \mathrm{m}^{2}, \text { mean } \\
(\mathrm{SD})\end{array}$ & $36.5(7.4)$ & $36.7(7.3)$ & - \\
\hline $\begin{array}{l}\text { HbAlc, \%, mean } \\
\quad(\mathrm{SD})\end{array}$ & $8.5(1.6)$ & $8.4(1.7)$ & 0.05 \\
\hline $\begin{array}{l}\text { CCI score, mean } \\
\text { (SD) }\end{array}$ & $0.5(0.9)$ & $0.5(1.0)$ & 0.01 \\
\hline \multicolumn{4}{|c|}{ Baseline antidiabetic medication, number of claims (\%) } \\
\hline Metformin & $634(45.5)$ & $\begin{array}{l}618 \\
\quad(47.8)\end{array}$ & -0.01 \\
\hline DPP-4is & $253(18.1)$ & $\begin{array}{l}237 \\
\quad(18.3)\end{array}$ & -0.02 \\
\hline SGLT-2is & $161(11.5)$ & $69(5.3)$ & - \\
\hline Sulfonylureas & $313(22.5)$ & $\begin{array}{l}302 \\
(23.4)\end{array}$ & 0.00 \\
\hline
\end{tabular}

$C C I$ Charlson Comorbidity Index, DPP-4i dipeptidyl peptidase-4 inhibitor, GLP-1 RA glucagon-like peptide-1 receptor agonist, $H b A 1 c$ glycated haemoglobin, $P S$ propensity score, $S D$ standard deviation, SGLT-2i sodium-glucose cotransporter-2 inhibitor; SMD standardized mean difference 
proportions of the patients in each cohort were women $(50 \% ; 51 \%)$, and similar proportions of the patients in the two groups had received metformin $(45 ; 48 \%)$, DPP-4is (18\%) and sulfonylureas $(22 \% ; 23 \%)$. There was a disparity in the proportions of patients who had received SGLT-2is $(12 \%$; 5\%). Cohorts were balanced with regard to SGLT-2i use $(8 \% ; 6 \%)$ when previous CVD and SGLT-2i use were included as a criterion in PS matching in a sensitivity analysis; the full baseline characteristics of patients included in this analysis are shown in Supplemental Table S4. Baseline characteristics for patients who were not included in the matched analyses are shown in Supplemental Table S5.

\section{Persistence in PS-Matched Cohorts}

The median stay time was 333 days for onceweekly GLP-1 RAs and 269 days for daily GLP-1 RAs. Once-weekly GLP-1 RAs were associated with significantly higher persistence than daily GLP-1 RAs over 12 months (once-weekly: 376/784 patients persistent [48\%]; daily: $322 / 784$ patients persistent [41\%]) and a $20 \%$ lower risk of treatment discontinuation (HR 0.80 [95\% confidence interval (CI) $0.71,0.90$ ], $p<0.01$; Fig. 2).

When the time that defines discontinuation was increased from $\geq 60$ to $\geq 90$ days not covered by medication for a sensitivity analysis, median stay time increased to 421 days for once-weekly GLP-1 RAs and to 324 days for daily GLP-1 RAs. In the sensitivity analysis that included previous CVD and SGLT-2i use in PS matching, the median stay time was unchanged for daily GLP-1 RAs (269 days) and slightly decreased for once-weekly GLP-1 RAs (323 days).

\section{Adherence in PS-Matched Cohorts}

Once-weekly GLP-1 RAs were associated with significantly higher adherence than daily GLP-1 RAs at 6 months (once-weekly: 427/784 patients adherent [54\%]; daily: 343/784 patients adherent [44\%]; $p<0.01)$.

At 12 months, adherence to once-weekly GLP-1 RAs was significantly higher than

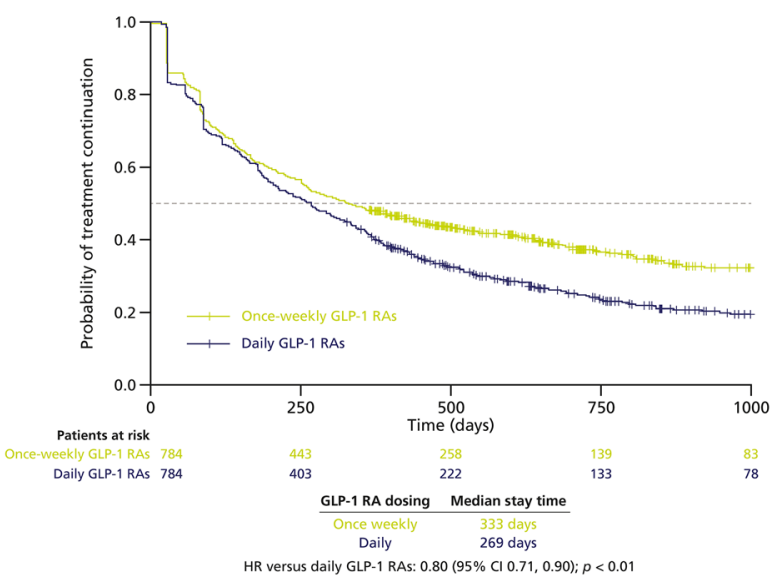

Fig. 2 Kaplan-Meier analysis of time to treatment discontinuation in patients treated with once-weekly vs daily injectable GLP-1 RAs (PS-matched cohorts). Dotted line represents median stay time. $C I$ confidence interval, $G L P-1 R A$ glucagon-like peptide-1 receptor agonist, $H R$ hazard ratio, $P S$ propensity score

adherence to daily GLP-1 RAs (once-weekly: 361/784 patients adherent [46\%]; daily: 263/784 patients adherent [34\%]; $p<0.01$ ). Percentages of adherent patients at 12 months remained the same in a sensitivity analysis that included previous CVD and SGLT-2i use in the PS matching.

\section{Change in HbA1c from Baseline in PS- Matched Cohorts}

Following treatment initiation with GLP-1 RAs, mean reductions in $\mathrm{HbA} 1 \mathrm{c}$ from baseline were greater with once-weekly than with daily GLP-1 RAs at 6 months and 12 months (Fig. 3). Regardless of adherence, improvements of - $1.1 \%(95 \%$ CI - 1.3, - 0.9) in the once-weekly GLP-1 RA cohort and $-0.9 \%$ (95\% CI -1.0 , - 0.7) in the daily GLP-1 RA cohort were observed at 6 months post-index (Fig. 3a); the difference between groups was statistically significant $(p<0.05)$. At 12 months post-index, a similar trend was noted, but the difference did not achieve statistical significance $(p>0.05$; Fig. 3b).

Greater adherence was associated with greater improvements in HbA1c levels for both treatment regimens. Mean reductions in HbA1c 

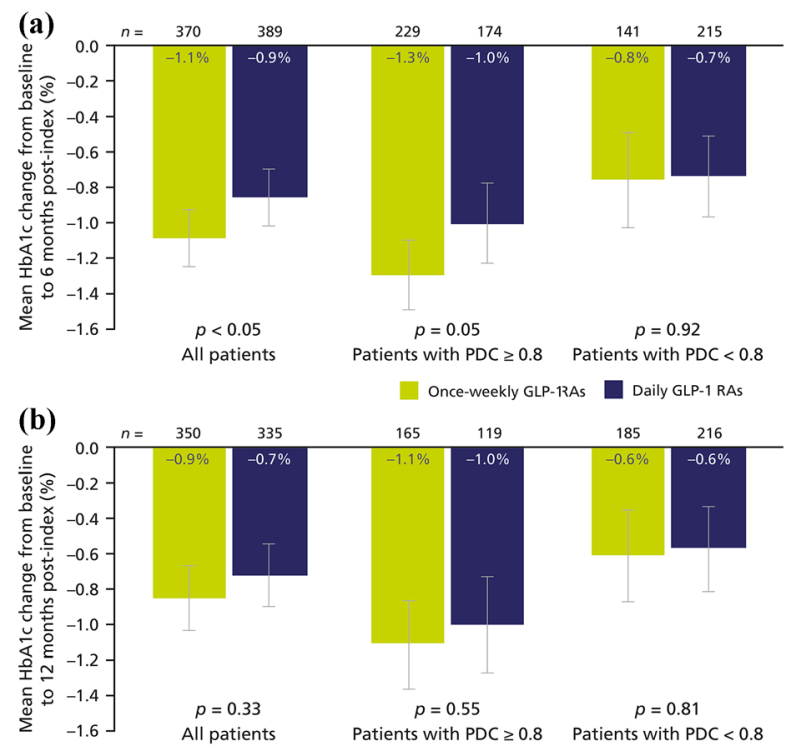

Fig. 3 Changes in $\mathrm{HbAlc}$ from baseline at 6 months postindex (a) and 12 months post-index (b) in PS-matched cohorts. Error bars represent $95 \%$ confidence intervals. Note that numbers in bars are rounded to one decimal place, whereas plotted data have two decimal places. GLP$1 R A$ glucagon-like peptide-1 receptor agonist, $H b A 1 c$ glycated haemoglobin, $P D C$ proportion of days covered, PS propensity score

were greater in adherent patients (PDC $\geq 0.8$ ) than in patients with PDC $<0.8$ over 6 months for both once-weekly GLP-1 RAs (- 1.3\% [95\% CI $-1.5,-1.1]$ vs $-0.8 \%[95 \%$ CI $-1.0,-0.5])$ and daily GLP-1 RAs (- 1.0\% [95\% CI -1.2 , - 0.8] vs - 0.7\% [95\% CI - 1.0, - 0.5]; Fig. 3a); the difference between the changes achieved with once-weekly and daily GLP-1 RAs did not reach statistical significance. Similar results were observed at 12 months (Fig. 3b).

\section{Change in Weight from Baseline in PS- Matched Cohorts}

The observed mean weight changes were clinically in the same range for both GLP-1 RA cohorts, but numerically higher at 6 months with daily regimens $(-2.5 \mathrm{~kg}$ [95\% CI -2.8 , -2.1]) than with once-weekly regimens $(-2.1 \mathrm{~kg}[95 \% \mathrm{CI}-2.6,-1.7] ; p>0.05$; Fig. $4 \mathrm{a})$. Weight loss was sustained over 12 months (daily GLP-1 RAs: $2.6 \mathrm{~kg}$ [95\% CI - 3.2, - 2.0]; once-weekly GLP-1 RAs: $2.0 \mathrm{~kg}$ [95\% CI -2.5 , - 1.5]; $p>0.05$; Fig. 4b).

Similar to trends observed with changes in HbA1c, weight reductions were more pronounced in adherent patients than in poorly adherent patients at 6 months and at 12 months with both once-weekly and daily GLP-1 RAs (Fig. 4). For example, after 12 months of treatment with once-weekly GLP1 RAs, a mean weight loss of $-3.1 \mathrm{~kg}(95 \% \mathrm{CI}$ $-3.9,-2.3$ ) was reported for adherent patients, whereas the mean weight loss in poorly adherent patients over the same time frame was $-1.1 \mathrm{~kg}$ (95\% CI - 1.8, - 0.4). The difference between the changes achieved with onceweekly and daily GLP-1 RAs did not reach statistical significance, except in patients with PDC $<0.8$ at 12 months $(p=0.02)$.

\section{Outcomes in IPTW-Matched Cohorts}

In the secondary analysis, the characteristics of patients receiving once-weekly or daily GLP-1 RAs were matched to those of basal insulin initiators. The baseline characteristics of the

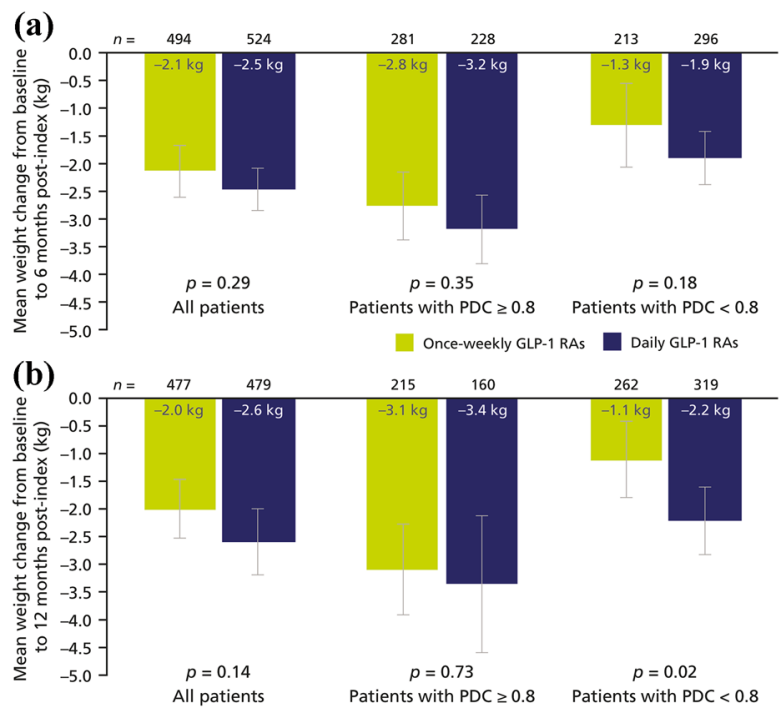

Fig. 4 Changes in weight from baseline at 6 months postindex (a) and 12 months post-index (b) in PS-matched cohorts. Error bars represent 95\% confidence intervals. Note that numbers in bars are rounded to one decimal place, whereas plotted data have two decimal places. GLP$1 R A$ glucagon-like peptide-1 receptor agonist, $P D C$ proportion of days covered, PS propensity score 
unmatched cohort of basal insulin users are shown in Supplemental Table S2, and the baseline characteristics of the GLP-1 RA cohorts after IPTW matching are shown in Supplemental Table S6. The groups of IPTW-matched onceweekly and daily GLP-1 RA users were well balanced, with SMDs vs insulin users $<0.1$ for all characteristics. Patients had a similar baseline mean age (once-weekly GLP-1 RAs: 58 years; daily GLP-1 RAs: 58 years), body mass index $\left(34 \mathrm{~kg} / \mathrm{m}^{2} ; 35 \mathrm{~kg} / \mathrm{m}^{2}\right)$ and CCI score $(0.7$ each). Similar proportions of patients in each cohort were women $(43 \% ; 48 \%)$, and use of antidiabetic medication at baseline was balanced across the two cohorts, except for SGLT-2i use.

Following IPTW matching to the characteristics of basal insulin users, the study results were consistent with those obtained after PS matching. Once-weekly GLP-1 RAs were associated with significantly higher persistence compared with daily GLP-1 RAs (median stay time: 276 days vs 213 days; HR $0.81 \quad[95 \%$ CI 0.77-0.85]; $p<0.01 ;$ Fig. 5).

Overall, $43 \%$ of patients receiving onceweekly GLP-1 RAs and 36\% of patients receiving daily GLP-1 RAs persisted with treatment for 12 months. Once-weekly GLP-1 RAs were associated with significantly higher adherence relative to daily GLP-1 RAs at 6 months $(51 \%$ vs $40 \% ; p<0.01)$ and at 12 months (43\% vs $31 \%$; $p<0.01)$.

Once-weekly GLP-1 RAs were also associated with significantly greater improvements in HbA1c compared with daily GLP-1 RAs at 6 months $(-2.0 \% \quad[95 \%$ CI $-2.2,-1.8]$ vs $-1.5 \%[-1.6,-1.3] ; p<0.01)$ and 12 months $(-1.9 \%[-2.2,-1.7]$ vs $-1.3 \%[-1.5,-1.1]$; $p<0.01)$; HbA1c reductions were greatest for adherent patients receiving once-weekly GLP-1 RAs (Supplemental Fig. S1).

Mean weight reductions in patients treated with daily GLP-1 RAs were greater than those associated with once-weekly GLP-1 RAs, although clinically similar at 6 months $(-2.0 \mathrm{~kg}$ [95\% CI - 2.4, - 1.7] vs $-1.6 \mathrm{~kg}[-2.1,-1.1]$; $p>0.05)$ and 12 months $(-2.3 \mathrm{~kg}[-2.8,-1.8]$ vs $-1.1 \mathrm{~kg}[-1.6,-0.6] ; p<0.01$; Supplemental Fig. S2). Again, better adherence was associated with greater weight reductions; this was

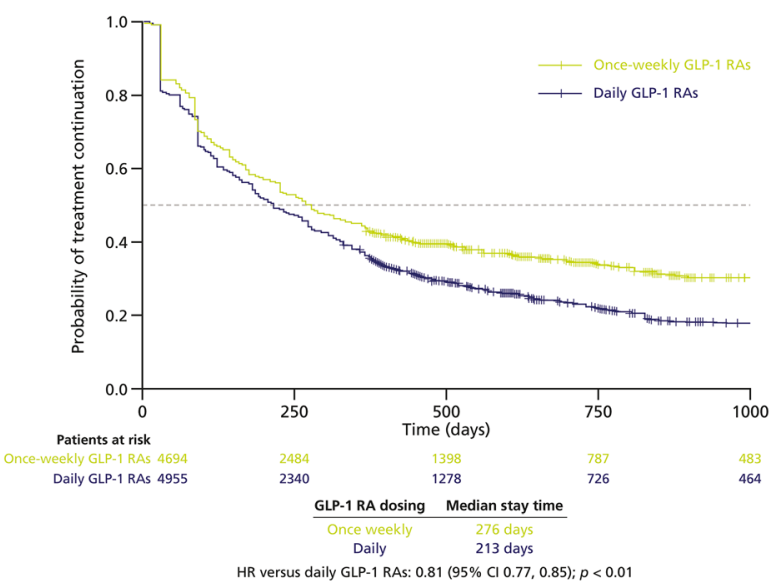

Fig. 5 Kaplan-Meier analysis of time to treatment discontinuation in patients treated with once-weekly vs daily injectable GLP-1 RAs (IPTW-matched cohorts). Dotted line represents median stay time. GLP-1 RA glucagon-like peptide-1 receptor agonist, IPTW inverse probability of treatment weighting

particularly evident for patients treated with once-weekly GLP-1 RAs at 12 months post-index, where a mean weight loss of $-2.8 \mathrm{~kg}(95 \%$ CI - 3.6, - 2.1) was reported for adherent patients, whereas poor adherence was associated with a weight gain of $0.2 \mathrm{~kg}(-0.5,0.9)$ (Supplemental Fig. S2). A statistically significant difference between treatment types was not found, except in patients with $\mathrm{PDC}<0.8$ at 12 months $(p<0.01)$.

\section{DISCUSSION}

This study aimed to characterize persistence and adherence in a large real-world population of patients with T2D treated with either onceweekly or daily GLP-1 RAs. Our results indicate that, compared with daily GLP-1 RAs, GLP-1 RAs administered once weekly are associated with significantly better persistence and greater adherence in patients treated in US clinical practice. This is in line with previous real-world studies reporting greater persistence and adherence for patients with T2D receiving treatment with once-weekly GLP-1 RAs rather than daily GLP-1 RAs. For example, in a study analysing data from a US administrative claims 
database, patients initiating once-weekly GLP-1 RAs had significantly higher adherence than patients on daily regimens at 12 months $(64 \%$ vs $44 \% ; p<0.001$ ) [22], which are higher rates than those reported in our study. However, it should be noted that this study did not match treatment groups by baseline characteristics, and therefore had a larger $(n=4791)$ and less selected patient population. In our study, a greater number of adherent patients may have been excluded from the matched analyses. Persistence and adherence rates similar to those seen in our study were reported in another US observational study that used PS-matched cohorts to compare outcomes in patients with T2D initiating treatment with once-weekly dulaglutide or with once-daily liraglutide [23]. The impact of dosing frequency on adherence was also investigated in a 2021 meta-analysis of seven real-world studies evaluating more than 75,000 patients with T2D. In this analysis, onceweekly GLP-1 RAs were associated with an 11\% lower risk of non-adherence compared with once-daily GLP-1 RAs (risk ratio: $0.89 ; I^{2}=89 \%$ ) [24]. It is important to note that, due to the timing of data collection, oral GLP-1 RAs were not included in our analyses. Persistence with and adherence to this newer formulation are likely to be different from the persistence and adherence observed with injectable GLP-1 RAs.

In a sensitivity analysis, previous CVD and SGLT-2i use were included in PS matching. Adherence and persistence results stayed the same even when previous CVD and SGLT-2i use were adjusted for. The cohorts were balanced across all baseline characteristics in this analysis, including SGLT-2i use (once-weekly GLP-1 RAs: 8\%; daily GLP-1 RAs: 6\%), for which there was a disparity in SGLT-2i use across PS-matched cohorts of the main analysis (once-weekly GLP-1 RAs: $12 \%$; daily GLP-1 RAs: $5 \%$ ). It is therefore plausible that this imbalance reflects an underlying difference in the proportion of patients with previous CVD events; however, it did not contribute to differences in persistence and adherence.

In our study, reductions in HbA1c were significantly greater at 6 months (but not at 12 months) in adherent patients compared with those poorly adherent to treatment. This suggests that, by improving patient adherence, less frequent dosing regimens may increase patients' chances of achieving glycaemic control and may improve their long-term outcomes. Response to treatment may in turn also impact on persistence and adherence, but cause and effect are difficult to separate. However, together with existing evidence of greater clinical effectiveness associated with better persistence and adherence [9], our results indicate that the use of more convenient treatment regimens may provide clinical as well as convenience benefits for patients with T2D. Furthermore, the use of once-weekly regimens requires fewer injections than daily regimens (52 vs 365 or more self-injections over the course of a year), which will also be of benefit to the environment.

In analyses that matched patients receiving GLP-1 RAs according to the clinical and demographic characteristics of basal insulin initiators, persistence and adherence results were similar to those of the main analysis. Selecting and matching to patients whose characteristics mimic those of people initiating insulin can provide a proxy for a "more progressed" cohort of patients with T2D. Thus, our results suggest that once-weekly regimens may also benefit patients whose T2D has progressed and who require treatment intensification with insulin for glycaemic control. This may indicate that medication schedules that improve persistence and adherence can provide benefits throughout the different stages of T2D progression. Convenience of medication is known to influence adherence [25]; by improving convenience and thereby adherence, for example via less frequent dosing, it may be possible not only to enhance glycaemic control but to improve quality of life [26, 27] and long-term health outcomes such as mortality [25] and to reduce healthcare costs [28]. Further research on reducing barriers to medication adherence and identifying the reasons for poor adherence and treatment discontinuation is merited.

The key strength of this study is the use of linked EHR and claims data, which allowed for a comprehensive analysis, connecting persistence and adherence to clinical outcomes. The large IBM MarketScan Explorys Claims-EMR Data Set 
captures relevant treatment use and outcomes, while the use of administrative claims data rather than prescription data means that persistence and adherence can be assessed accurately, because claims can provide robust estimates of how patients took their medication [13]. The retrospective, observational nature of this study introduces a risk of bias; however, use of PS matching limited bias in this study and allowed a comparison of clinically relevant changes between treatment groups with balanced baseline characteristics. In addition, the key results of this study were confirmed using IPTW as an alternative way of matching patients.

Despite our use of a large, comprehensive data source, this study had some limitations. A sizeable proportion of the eligible patients in the database could not be PS matched owing to missing HbA1c and weight data and were therefore not included in the analysis. Due to the resulting number of PS-matched patients $(n=784)$, persistence and adherence could not be evaluated for individual GLP-1 RAs. A further limitation is that reasons for discontinuation could not be assessed because of the retrospective nature of this study. For example, we suspect that if the analyses could have been limited to those who had chosen on their own to discontinue (i.e. were not recommended by their clinician to discontinue or switch to another medication), the benefits of persistence and adherence in our study would have been even more apparent.

\section{CONCLUSION}

Our results suggest that, in a real-world setting, once-weekly injectable GLP-1 RAs are associated with better persistence and adherence than daily regimens over 1 year. Better adherence to treatment was associated with greater reductions in HbA1c levels for both once-weekly and daily regimens. Our study provides evidence that persistence and adherence, which are typically considered to be linked to patient convenience, also have clear clinical benefits. Improving persistence with and adherence to medication among patients with T2D should be a key objective for healthcare systems, payers and policy makers.

\section{ACKNOWLEDGEMENTS}

Funding. This study, the graphical abstract and the Rapid Service Fee for publication, was funded by Novo Nordisk A/S.

Medical Writing Assistance. Medical writing assistance in the preparation of this article was provided by Oxford PharmaGenesis, Oxford, UK, and funded by Novo Nordisk.

Authorship. All named authors meet the International Committee of Medical Journal Editors (ICMJE) criteria for authorship for this article, take responsibility for the integrity of the work as a whole, and have given their approval for this version to be published.

Author Contributions. Conceptualization: Riya Arora, João Fernandes; Methodology: Riya Arora, Mads Faurby, João Fernandes, Andreas Liebl, William H. Polonsky; Writing-review and editing: Riya Arora, Mads Faurby, João Fernandes, Andreas Liebl, William H. Polonsky.

Disclosures. William H. Polonsky has served as a consultant for Eli Lilly, Novo Nordisk and Sanofi. Riya Arora is an employee of Novo Nordisk Service Centre India Pvt Ltd, which is part of Novo Nordisk A/S. Mads Faurby is an employee of Novo Nordisk A/S. João Fernandes was an employee of Novo Nordisk A/S at the time of the analysis, and is now an employee of Ferring Pharmaceuticals A/S. Andreas Liebl has received research support and honoraria for giving presentations and attending advisory boards from AstraZeneca, Bayer, Becton Dickinson, Boehringer Ingelheim, Bristol Myers Squibb, Eli Lilly, Medtronic, MSD, Novo Nordisk, Roche and Sanofi.

Compliance with Ethics Guidelines. Approval by an ethics committee was not required for this study, because only de-identified secondary data were used. The IBM MarketScan 
Explorys Claims-EMR Data Set (IBM Watson Health, Armonk, NY, USA) is a commercial database and the datasets were accessed through a licence with IBM. The study was performed in accordance with the Helsinki Declaration of 1964 and its later amendments.

Data Availability. Data supporting the findings of this study are not publicly available because they are under licence from IBM. Aggregate datasets generated during and analysed during the current study are available from the corresponding author on reasonable request.

Open Access. This article is licensed under a Creative Commons Attribution-NonCommercial 4.0 International License, which permits any non-commercial use, sharing, adaptation, distribution and reproduction in any medium or format, as long as you give appropriate credit to the original author(s) and the source, provide a link to the Creative Commons licence, and indicate if changes were made. The images or other third party material in this article are included in the article's Creative Commons licence, unless indicated otherwise in a credit line to the material. If material is not included in the article's Creative Commons licence and your intended use is not permitted by statutory regulation or exceeds the permitted use, you will need to obtain permission directly from the copyright holder. To view a copy of this licence, visit http://creativecommons.org/licenses/by$\mathrm{nc} / 4.0 /$.

\section{REFERENCES}

1. Tran KL, Park YI, Pandya S, et al. Overview of glucagon-like peptide-1 receptor agonists for the treatment of patients with type 2 diabetes. Am Health Drug Benefits. 2017;10:178-88.

2. American Diabetes Association. Pharmacologic approaches to glycemic treatment: standards of medical care in diabetes-2021. Diabetes Care. 2021;44:S111-24.

3. Food and Drug Administration. Highlights of prescribing information. Rybelsus (semaglutide) tablets, for oral use. Revised: 2019. https://www. accessdata.fda.gov/drugsatfda_docs/label/2019/ 213051s000lbl.pdf. Accessed 18 May 2021.

4. European Medicines Agency. Rybelsus. Summary of product characteristics. 2020. https://www.ema. europa.eu/en/documents/product-information/ rybelsus-epar-product-information_en.pdf. Accessed 18 May 2021.

5. Gentilella R, Pechtner V, Corcos A, Consoli A. Glucagon-like peptide- 1 receptor agonists in type 2 diabetes treatment: are they all the same? Diabetes Metab Res Rev. 2019;35:e3070.

6. Vogler S, Haasis MA, Dedet G, Lam J, Bak Pedersen $\mathrm{H}$. Medicines reimbursement policies in Europe. WHO Regional Office for Europe. 2018. https:// www.euro.who.int/_data/assets/pdf_file/0011/ 376625/pharmaceutical-reimbursement-eng.pdf. Accessed 9 Jun 2021.

7. Medicine Matters Diabetes. A quick guide to the SUSTAIN trials. Updated January 2021. 2017. https://diabetes.medicinematters.com/en-GB/ semaglutide/type-2-diabetes/a-quick-guide-to-thesustain-trials/12206922. Accessed 4 Jun 2021.

8. Nauck MA. The design of the liraglutide clinical trial programme. Diabetes Obes Metab. 2012;14: 4-12.

9. Guerci B, Chanan N, Kaur S, Jasso-Mosqueda JG, Lew E. Lack of treatment persistence and treatment nonadherence as barriers to glycaemic control in patients with type 2 diabetes. Diabetes Ther. 2019;10:437-49.

10. Claxton AJ, Cramer J, Pierce C. A systematic review of the associations between dose regimens and medication compliance. Clin Ther. 2001;23: 1296-310.

11. Divino V, DeKoven M, Khan FA, et al. GLP-1 RA treatment patterns among type 2 diabetes patients in five European countries. Diabetes Ther. 2017;8: $115-28$.

12. IBM. Explorys EHR solutions. https://www.ibm. com/products/explorys-ehr-data-analysis-tools/ details. Accessed 4 May 2021.

13. IBM Watson Health. White paper: IBM MarketScan research databases for life sciences researchers 2021. https://www.ibm.com/downloads/cas/ OWZWJ0QO. Accessed 10 Nov 2021.

14. Aroda VR, Faurby M, Lophaven S, et al. Insights into the early use of oral semaglutide in routine clinical practice: the IGNITE study. Diabetes Obes Metab. 2021;23:2177-82. 
15. Sullivan SD, Bailey TS, Roussel R, et al. Clinical outcomes in real-world patients with type 2 diabetes switching from first- to second-generation basal insulin analogues: comparative effectiveness of insulin glargine 300 units $/ \mathrm{mL}$ and insulin degludec in the DELIVER D+ cohort study. Diabetes Obes Metab. 2018;20:2148-58.

16. ICD.Codes. ICD-9-CM chapters. 2016. https://icd. codes/icd9cm. Accessed 17 Jun 2021.

17. ICD.Codes. ICD-10-CM chapters. 2016. https://icd. codes/icd10cm. Accessed 17 Jun 2021.

18. Austin PC. An introduction to propensity score methods for reducing the effects of confounding in observational studies. Multivariate Behav Res. 2011;46:399-424.

19. Austin PC, Stuart EA. Moving towards best practice when using inverse probability of treatment weighting (IPTW) using the propensity score to estimate causal treatment effects in observational studies. Stat Med. 2015;34:3661-79.

20. Heinze G, Jüni P. An overview of the objectives of and the approaches to propensity score analyses. Eur Heart J. 2011;32:1704-8.

21. Rudolph KE, Colson KE, Stuart EA, Ahern J. Optimally combining propensity score subclasses. Stat Med. 2016;35:4937-47.

22. Weiss T, Carr RD, Pal S, et al. Real-world adherence and discontinuation of glucagon-like peptide-1 receptor agonists therapy in type 2 diabetes mellitus patients in the United States. Patient Prefer Adherence. 2020;14:2337-45.

23. Mody R, Huang Q, Yu M, et al. Adherence, persistence, glycaemic control and costs among patients with type 2 diabetes initiating dulaglutide compared with liraglutide or exenatide once weekly at 12-month follow-up in a real-world setting in the United States. Diabetes Obes Metab. 2019;21:920-9.

24. Weeda ER, Muraoka AK, Brock MD, Cannon JM. Medication adherence to injectable glucagon-like peptide-1 (GLP-1) receptor agonists dosed once weekly vs once daily in patients with type 2 diabetes: a meta-analysis. Int J Clin Pract. 2021;75: e14060.

25. Polonsky WH, Henry RR. Poor medication adherence in type 2 diabetes: recognizing the scope of the problem and its key contributors. Patient Prefer Adherence. 2016;10:1299-307.

26. Evans $\mathrm{M}$, Jensen $\mathrm{HH}$, Bøgelund $\mathrm{M}$, et al. Flexible insulin dosing improves health-related quality-oflife (HRQoL): a time trade-off survey. J Med Econ. 2013;16:1357-65.

27. Boye KS, Matza LS, Walter KN, et al. Utilities and disutilities for attributes of injectable treatments for type 2 diabetes. Eur J Health Econ. 2011;12:219-30.

28. Jha AK, Aubert RE, Yao J, Teagarden JR, Epstein RS. Greater adherence to diabetes drugs is linked to less hospital use and could save nearly $\$ 5$ billion annually. Health Aff. 2012;31:1836-46. 